\title{
Envolvimento dos Utilizadores na definição de Requisitos: o caso da plataforma WEBMAT
}

\author{
António Pedro Costa ${ }^{1}$, Estela Barreto Coelho ${ }^{2}$, Liliana Cristina Tavares ${ }^{3}$ e Fábio Freitas ${ }^{4}$ \\ apcosta@ua.pt, estelacoelho@gmail.com, ltavares@esec.pt, fabiomauro@ua.pt
}

${ }^{1}$ Ludomedia e CIDTFF - Centro de Investigação Didáctica e Tecnologia na Formação de Formadores DE/UADepartamento de Educação, Universidade de Aveiro, Aveiro, Portugal

${ }^{2}$ Agrupamento de Escolas de Vale de Ovil - Baião, Oliveira de Azeméis, Portugal

${ }^{3}$ Escola Superior de Educação de Coimbra, Instituto Politécnico de Coimbra, Coimbra, Portugal

${ }^{4}$ CIDTFF - Centro de Investigação Didáctica e Tecnologia na Formação de Formadores DE/UA-

Departamento de Educação, Universidade de Aveiro, Aveiro, Portugal

DOI: 10.17013/risti.e4.92-106

Resumo: Há alguns anos a esta parte, o ensino e a aprendizagem da Matemática estão sujeitos a um intenso processo de inovação e de reestruturação, não apenas ao nível dos conteúdos e metas, mas também, e especialmente, ao nível das estratégias a implementar na lecionação desses mesmos conteúdos. Por outro lado, a realidade atual evidencia a importância que os artefactos tecnológicos propiciam na definição dessas estratégias. Neste âmbito, surgiu a ideia de desenvolver uma plataforma designada por WEBMAT - Materiais Manipuláveis, que irá consistir numa ferramenta em que os utilizadores concebem as suas propostas de trabalho na área da matemática tendo por base Materiais Manipuláveis (por exemplo, blocos lógicos, cuisenaire, blocos geométricos, entre outros). A plataforma terá como público-alvo, docentes e alunos do pré-escolar e $1^{\circ}$ ciclo. Os resultados obtidos através da aplicação de um inquérito por questionário reforçam a importância de envolver o utilizador nas fases iniciais da definição de requisitos.

Palavras Chave: Materiais Manipuláveis; Software Educativo; Design Centrado no Utilizador, Envolvimento do Utilizador.

\section{Users involvement in defining of requirements: the case of WEBMAT plataform}

Abstract: A few years now, mathematics teaching and learning are subject to an intensive process of innovation and restructuring, not only in terms of content and goals, but also, and especially, in terms of the strategies to implement the teaching of these same contents. On the other hand, the current situation highlights the importance that technological artefacts provide in defining these strategies. In this context, came the idea of developing a platform called WEBMAT - manipulatives, which will consist of a tool where users design their work proposals in the area of mathematics based on manipulatives (eg, logic blocks, cuisenaire rods, geometric 
blocks). The platform will have as target, teachers and students from kindergarten and $1^{\text {st }}$ cycle. The results obtained by applying a questionnaire survey reinforce the importance of involving the user in the early stages of requirements definition.

Keywords: Manipulatives; Educational Software; User Centered Design, User Involvement.

\section{Introdução}

Lançando um breve olhar sobre o passado do ensino e aprendizagem da Matemática, verifica-se que os conteúdos desta disciplina eram apresentados pelo Professor, aos aprendentes, de uma forma direta e demonstrativa. Pretendia-se que os segundos memorizassem noções, definições e conceitos e que treinassem, repetidamente, técnicas e procedimentos, sem compreenderem, muitas vezes, os conteúdos e a sua aplicabilidade (Mizukami, 1986).

Acreditando na premissa que a aprendizagem deve ser feita de forma construtiva, deve-se colocar o aluno num ambiente em que ele possa ser capaz de construir, autonomamente, os seus próprios conhecimentos. Este estilo "Convergente" de aprendizagem, enquadrase na teoria experimental de aprendizagem defendida por David Kolb (1984), na qual o autor define um modelo de quatro estilos de aprendizagem: i) Divergência; ii) Assimilação; iii) Convergência; e iv) Acomodação. O estilo "Convergente" de aprendizagem é caracterizado por indivíduos que apreciam tarefas técnicas, onde possam aprender experimentando novos conceitos, simulando e testando na prática a sua própria aprendizagem.

Dado este cenário, a instituição Escola não pode ficar apática perante este desafio, nem perante os novos métodos e técnicas que foram surgindo e que contribuem largamente para que este fim seja alcançado com sucesso. As tecnologias de informação e de comunicação e, especificamente, os pacotes de software educativo elegem-se, hoje, como os principais atores nestes novos processos metodológicos. Esta ideia é suportada pelo Horizon Report Europe (Johnson et al., 2014), ao referir que a aprendizagem por meio de jogos de computadores e a congregação de métodos de ensino tradicional e virtual, deverão integrar o ensino nos próximos dois a três anos. O mesmo relatório insta as escolas a adotarem medidas urgentes que promovam a inovação nas salas de aula, de forma a tirar proveito de uma maior utilização das redes sociais e dos recursos educativos abertos.

Na atualidade, e conforme refere o Programa e as Metas Curriculares de Matemática do Ensino Básico, defende-se a criação de práticas e estruturas no ensino da matemática que permitam desenvolver conhecimentos, capacidades e atitudes de forma integrada.

O ensino da Matemática não deverá, portanto, adicionar somente capacidades de resolução de problemas, raciocínio e comunicação, mas também atitudes favoráveis à atividade matemática, a um currículo baseado em conhecimentos isolados e técnicas de cálculo (Bivar, Grosso, Oliveira, \& Timóteo, 2013).

Pretende-se que o Professor auxilie o aluno a construir o seu conhecimento matemático, ou seja, que o Professor coloque o aluno numa posição em que este consiga, por si só e ao seu ritmo, desenvolver aprendizagens baseadas no concreto, raciocínios matemáticos, 
conjunturas de conhecimentos, atitudes, capacidades e competências, transformandose assim num ser matematicamente competente.

Para que o aluno consiga assumir esta dimensão construtivista por inteiro e consequentemente adquira o seu conhecimento a partir das suas ações, o Professor deve propiciar-lhe recursos, bem como experiências significativas e variadas tais como, materiais didáticos (manipuláveis), recursos audiovisuais e aplicações tecnológicas, problemas concretos e relacionados com o quotidiano, atividades de investigação, projetos e jogos (Bivar et al., 2013). Neste âmbito, a plataforma WEBMAT apresenta conteúdos concebidos para explorar conceitos matemáticos mas, mais do que seguir esta linha de "conteúdo feito", a WEBMAT irá apresentar-se como uma ferramenta de autoria. O utilizador constrói as suas propostas de trabalho. Os tópicos/temas matemáticos explorados no desenvolvimento das tarefas são definidos pelo utilizador, que poderá guardar as suas tarefas online, permitindo-lhe aceder às mesmas com facilidade e em qualquer lugar.

Após estas considerações iniciais, importa perceber o conteúdo das secções seguintes do presente artigo. Assim a próxima secção apresenta a importância do uso dos materiais manipuláveis e do uso das tecnologias de informação e comunicação nos primeiros anos de escolaridade. Na terceira secção evidencia-se a importância do envolvimento do utilizador no processo de desenvolvimento da plataforma WEBMAT. Por sua vez, a quarta secção apresenta o processo metodológico e a quinta secção a análise e discussão dos resultados extraídos da aplicação do questionário. Por fim, surgem as conclusões deste estudo.

\section{Materiais Manipuláveis}

Há muito se tem verificado que a mera reprodução de tarefas e exercícios matemáticos em nada contribuí para a aprendizagem e o desenvolvimento das crianças, tornando-as cada vez mais desinteressadas e desmotivadas perante a disciplina (Ponte, 2003). Segundo o estudo realizado pela UNESCO (2014), cabe ao Professor contrariar este cenário, procurando proporcionar experiências ricas e desafiadoras, grandes momentos de construção de saberes, utilizando os conhecimentos prévios dos alunos e as experiências do quotidiano, enquanto momentos propícios para a construção do saber Matemático e a solução de problemas. Existe um vasto número de materiais pedagógicos, tecnologias e metodologias capazes de fazer com que o ensino e a aprendizagem sejam um processo contínuo de construção e de descobertas de novos conceitos. Os materiais manipuláveis, levam os alunos a estabelecerem relações, inferências, aplicando e transformando o conhecimento já adquirido anteriormente, transpondo os saberes escolares para a vida quotidiana e vice-versa.

Parece relevante "equipar" as aulas de Matemática com todo um conjunto de materiais manipuláveis (cubos, geoplanos, tangrans, réguas, papel ponteado, ábaco, e tantos outros) e de material tecnológico (calculadora, computador, software educativo, etc.), em adequação com os problemas a resolver, as ideias a explorar ou estruturados de acordo com determinado conceito matemático.

Ainda no que concerne à inclusão do computador (e dispositivos móveis) nas aulas de Matemática e dado que cada vez mais vivemos em "ambientes digitais", este assume 
um papel essencial, como instrumento no desenvolvimento de experiências e no ensaio de estratégias de resolução de problemas. Mas, mais do que isso, ele é importante na construção da própria Matemática: na formulação, investigação e exploração de situações problemáticas, bem como no desenvolvimento do gosto pela disciplina.

\section{Envolvimento do Utilizador no Desenvolvimento de Software}

É pertinente que, no que toca a desenvolvimento de software, as empresas se consciencializem da importância de definir e padronizar conhecimentos, objetivos e métodos e apoiando a sua consolidação (Razón et al., 2014). Nesse sentido, a adoção de abordagens teóricas como a Usabilidade e a Experiência de Utilizador (ISO9241, 2010), o Design Centrado no Utilizador (Mao, Vredenburg, Smith, \& Carey, 2001; Marcus, 2005), o Design Participativo (Abras, Maloney-krichmar, \& Preece, 2004) e o Design Centrado no Aprendente (Soloway, Guzdial, \& Hay, 1994) identificam as potencialidades e os constrangimentos do envolvimento dos utilizadores nos processos de desenvolvimento de software. As mesmas abordagens descrevem como os utilizadores podem participar no processo de desenvolvimento de um software.

\subsection{Usabilidade e Experiência de Utilizador}

A integração das ferramentas digitais no contexto educativo é uma realidade cada vez mais presente, o que torna apropriada uma reflexão sobre a sua eficácia na aprendizagem, assim como na interação que as mesmas proporcionam aos estudantes e professores. Nesse sentido, torna-se imperativo que conceitos como Usabilidade e Experiência de Utilizador (User Experience - UX) sejam tidos em conta no envolvimento do utilizador no desenvolvimento de software.

Têm sido patentes alguns obstáctulos que a introdução de ferramentas tecnológicas têm trazido para os estudantes, como por exemplo, no concerne à leitura e compreensão de dados (Víctor López Simó \& Casulleras, 2013). É com a propósito de minimizar essas mesmas dificuldades, que os critérios de Usabilidade têm sido um foco e uma tendência no que se refere ao desenvolvimento de tecnologia relacionada com o Human Computer Interaction (HCI) (Scanlon, Mcandrew, \& Shea, 2015). Esse cuidado tem sido manifestado igualmente na elaboração de estudos que apoiem empresas didáticas a compreender como tornar os seus artigos mais eficientes e compreensíveis para o utilizador, recorrendo para tal a uma avaliação de Usabilidade e Experiência de Utilizador dos seus produtos (Freitas, Costa, \& Souza, 2015).

A Usabilidade apresenta-se como a vertente mais "racional" de um produto, facultando aos utilizadores o alcance de objetivos específicos de maneira eficiente e satisfatória (ISO9241, 2010). Por outro lado, a Experiência de Utilizador é em grande medida, o feedback proporcionado pela Usabilidade de um sistema, refletindo o lado mais "emocional" na utilização de um produto. Está relacionada com as preferências, perceções, emoções, crenças, reações físicas, e psicológicas por parte do utilizador no decorrer da utilização de um produto (ISO9241, 2010). Dessa forma, compreendese o prazer que muitas interfaces proporcionam aos utilizadores, o que evidencia a eficiência da integração dos conceitos de Usabilidade e Experiência de Utilizador no desenvolvimento de soluções de HCI (Scanlon et al., 2015). 


\subsection{Design Centrado no Utilizador}

No seguimento do Design Centrado no Utilizador (DCU) surgiram outras abordagens teóricas ao desenvolvimento de software como é caso do Design Centrado no Aprendente. Esta abordagem assume que todos somos aprendentes, independentemente de ser um profissional ou um aluno. Portanto, o foco principal do Design Centrado no Aprendente é garantir que o design de interface é apropriado aos interesses, conhecimentos e estilos dos aprendentes que utilizam o software (Nesset \& Large, 2004).

O envolvimento de crianças e de professores no processo de desenvolvimento de um software é um princípio fundamental da abordagem do DCU (Pardo, Vetere, \& Howard, 2005). O envolvimento dos utilizadores no processo de desenvolvimento providencia uma fonte de conhecimento sobre o contexto de utilização, sobre as tarefas e como os utilizadores tendem a trabalhar posteriormente com o software.

O grau de envolvimento dos utilizadores poderá variar consoante o papel e as tarefas a realizar. Os utilizadores podem assumir papéis menos interventivos, como o de verificadores (testers), como papéis mais interventivos, como o de informadores (Nesset \& Large, 2004) ou codesigners em que, os utilizadores designados como codesigners, são encarados como membros da equipa multidisciplinar (Preece, Rogers, \& Sharp, 2002).

A Tabela 1 apresenta algumas propostas de envolvimento dos utilizadores, em que fase do processo de desenvolvimento deverá ocorrer e que técnicas poderão ser utilizadas (Preece et al., 2002).

\begin{tabular}{lll}
\hline Proposta & Fase do Projeto & Através de... (técnicas) \\
\hline $\begin{array}{l}\text { Recolha de dados sobre as necessidades e } \\
\text { expetativas dos utilizadores; } \\
\begin{array}{l}\text { Avaliação de alternativas do projeto, } \\
\text { protótipos e produto final. }\end{array}\end{array}$ & $\begin{array}{l}\text { No início do } \\
\text { desenvolvimento }\end{array}$ & Entrevistas e Questionários \\
\hline $\begin{array}{l}\text { Recolha de dados relacionados com a } \\
\text { sequência de tarefas a ser realizada com o } \\
\text { software. }\end{array}$ & Fase inicial & Entrevistas e Questionários \\
\hline $\begin{array}{l}\text { Inclusão das partes interessadas para } \\
\text { discutir os requisitos do utilizador. }\end{array}$ & Fase inicial & Focus Groups \\
\hline $\begin{array}{l}\text { Recolha de dados sobre o contexto em que } \\
\text { será utilizado o software. }\end{array}$ & Fase inicial & Observação no local \\
\hline $\begin{array}{l}\text { Avaliação de soluções alternativas e } \\
\text { recolha de informações adicionais sobre as } \\
\text { necessidades e expetativas dos utilizadores. } \\
\text { Avaliação de protótipos. }\end{array}$ & $\begin{array}{l}\text { Fase inicial e } \\
\text { intermédia }\end{array}$ & $\begin{array}{l}\text { Jogo de papéis, walkthroughs, } \\
\text { simulações e maquetas }\end{array}$ \\
\hline $\begin{array}{l}\text { Recolha de dados quantitativos } \\
\text { transmitidos por critérios de usabilidade. }\end{array}$ & Fase final & Testes de usabilidade \\
\hline $\begin{array}{l}\text { Recolha de dados qualitativos transmitidos } \\
\text { pela satisfação dos utilizadores com a } \\
\text { utilização do software. }\end{array}$ & Fase final & Entrevistas e Questionários \\
\hline
\end{tabular}

Tabela 1 - Envolvimento dos Utilizadores vs Fase do Projeto 
Neste estudo e de acordo com a Tabela 1 focamos na recolha de dados sobre as necessidades e expetativas dos utilizadores, através da aplicação de um inquérito por questionário.

\subsection{Conceber para Professores e Professores como Codesigners}

Quando falamos do envolvimento do professor, surge-nos na literatura uma variação do Design Centrado no Utilizador designada por Design Centrado no Aprendente que, por sua vez está dividida em duas abordagens muito similares: i) Design Centrado no Currículo e o ii) Design Centrado na Sala de Aula. Ambas, destacam o contexto onde aprendizagem ocorre, incluindo os professores, as crianças, o ambiente físico e cultural. Em ambas, o processo de desenvolvimento é centrado nas aulas, sendo o papel do professor fundamental (Pardo et al., 2005).

O professor durante o processo de desenvolvimento do software pode desempenhar diferentes papéis, desde utilizador, em que é observado em atividades dinamizadas em contexto de sala de aula mediadas pela tecnologia ou como verificador ou informador sobre as suas práticas de ensino e o seu impacto no desempenho dos alunos. Nestes métodos, os alunos são envolvidos apenas como utilizadores e verificadores, uma vez que as suas necessidades como alunos são definidas pelo Professor e pelo currículo (Pardo et al., 2005).

A Figura 1 apresenta os quatros papéis que o Professor poderá representar (em relação às crianças) ao envolver-se no desenvolvimento de um software (Pardo et al., 2005; Pardo, Vetere, \& Howard, 2006):

- Facilitador: os Professores são os responsáveis por agendar sessões de trabalho durante o processo de desenvolvimento. Desta forma a interferência com as atividades de sala de aula e curriculares são reduzidas. São os Professores, enquanto facilitadores, que escolhem as crianças que irão participar durante o processo desenvolvimento do software e auxiliar os investigadores a formar os grupos de crianças quando necessário (Africano, et al., 2004; Theng, MohdNasir, Thimbleby, Buchanan, \& Jones, 2000);

- Verificadores (testers): os Professores avaliam as soluções de software existentes de forma a estimar o potencial valor dos mesmos e avaliar protótipos e produtos em desenvolvimento. Sendo os Professores verificadores, a equipa de desenvolvimento pode recolher dados sobre as suas práticas e experiências relativamente à utilização de outros recursos de apoio ao ensino (Pardo, et al., 2005);

- Informadores: os Professores são vistos como tendo uma visão incomparável sobre as práticas de aprendizagem e ensino, a que os elementos da equipa de desenvolvimento recorrem (Scaife, Rogers, Aldrich, \& Davies, 1997). A equipa procura recolher perceções sobre variadas áreas relativas à conceção e desenvolvimento de novas tecnologias. Pode-se solicitar que descrevam o uso dos computadores na sua escola, de forma a ajudar a definir as metas de aprendizagem, a identificar as dificuldades das crianças e as suas capacidades. Os Professores podem efetuar observações ou comentários quanto à conformidade das tarefas de aprendizagem ou atividades em que as crianças irão participar (nas sessões de teste ou validação), em termos de clareza e adequação tendo em conta as capacidades dos alunos (Africano et al., 2004); 
- Parceiros de Investigação: os Professores podem recolher dados para a equipa de desenvolvimento, sendo o processo facilitado pela sua experiência de contexto de sala de aula e familiaridade com as crianças ou podem dar apoio às crianças durantes as sessões de desenvolvimento ("The Role of Children in the Design of New Technology ," 2002). Na realidade os Professores ocupam uma posição privilegiada, pois diariamente interagem com as crianças e observandoas a interagir entre si, o que permite capturar dados no decorrer do tempo e durante diferentes atividades.

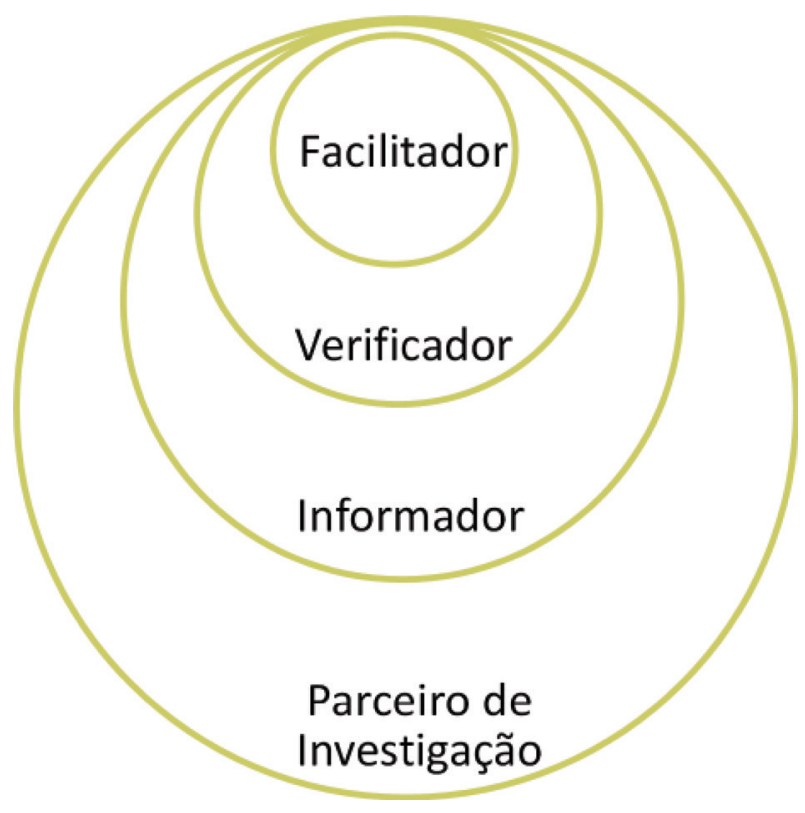

Figura 1 - Papéis do Professor no processo de desenvolvimento, adaptado de Pardo, Vetere \& Howard (2005)

\section{Processo Metodológico}

O desenvolvimento da plataforma WEBMAT assenta nos princípios definidos pela Metodologia Hibrida de Desenvolvimento Centrado no Utilizador (MHDCU) preconizada por Costa e colaboradores associados (A. P. Costa, Reis, \& Loureiro, 2014b). Sempre que é aplicada a MHDUC ao desenvolvimento de um software, os seus processos são melhorados através da análise: a) Comunicação; b) Coordenação; e Colaboração e Cooperação definidas no Modelo 4C (A. P. Costa, Reis, \& Loureiro, 2014, 2014a).

Para a recolha de dados foi disponibilizado um inquérito por questionário dividido em três grupos: a) Caraterização; b) Levantamento de Perceções Técnicas e Didáticas; e c) Sugestões. Pretendia-se assim perceber e analisar quais os requisitos que os utilizadores propunham para o desenvolvimento da plataforma WEBMAT. O questionário distribuído pela base de dados Ludomedia (dirigida a Educadores de Infância, Professores do $1^{\mathrm{o}}$ CEB e Professores de Educação Especial), rececionou 94 respostas. 
O questionário foi aplicado com intuito da equipa multidisciplinar tomar decisões de acordo com Costa (2012) quando afirma que "o envolvimento dos utilizadores no processo de desenvolvimento providencia uma fonte de conhecimento sobre o contexto de utilização, as tarefas, e a forma como os utilizadores tendem a trabalhar posteriormente com o software" (p. 182).

Para análise dos dados foi usado o software de apoio à análise qualitativa webQDA (Souza, Costa, \& Moreira, 2011). Como técnica foi aplicada a Análise de Conteúdo e de acordo com Robert e Bouillaguet (Robert \& Bouillaguet, 1997) esta “define-se como uma técnica que possibilita o exame metódico, sistemático, objetivo e, em determinadas ocasiões, quantitativo, do conteúdo de certos textos, com vista a classificar e a interpretar os seus elementos constitutivos e que não são totalmente acessíveis à leitura imediata" (p. 4).

Neste estudo de natureza mista o inquérito por questionário é constituído por nove questões, sendo cinco de carácter fechado e quatro de carácter aberto. As primeiras questões são direcionadas para a caracterização dos participantes: a) situação profissional; b) práticas profissionais referentes à sua formação académica, experiência profissional e quanto ao conhecimento e utilização de materiais manipuláveis nas suas práticas pedagógicas dentro e fora da sala de aula. As questões abertas: a) perceções técnicas; b) perceções sobre os conteúdos e atividades e; c) sugestões.

Na análise e discussão de resultados, referida na próxima secção do presente artigo, a equipa pretendeu responder à questão "Quais as atividades e conteúdos matemáticos que uma plataforma tecnológica deve permitir conceber?”.

\section{Análise e Discussão dos Resultados}

Apesar do estudo contemplar o levantamento de perceções técnicas, neste artigo apenas analisamos os dados referentes às perceções dos conteúdos e das atividades por parte dos Professores.

\subsection{Caracterização}

Obteve-se um total de 94 respostas. A maioria dos inquiridos, 73\%, possui Licenciatura, 25\% possui Mestrado e 2\% possui Doutoramento. Dos inquiridos, 46\% são Educadores de Infância, 31\% são Professores do 1. ${ }^{\circ} \mathrm{CEB}, 10 \%$ são Professores de Educação Especial e $13 \%$ exercem outra profissão.

Relativamente ao tempo de experiência (Fig. 2) no ensino, 92\% exerce a sua profissão há mais de 5 anos, 4\% exerce a sua profissão há mais de 3 anos e menos de 5 anos e por último, 4\% exerce a sua profissão há menos de 3 anos. $24 \%$ dos inquiridos utiliza os materiais manipuláveis todos os dias, 36\% utiliza entre 2 a 3 vezes por semana, $27 \%$ utiliza apenas uma vez por semana e $13 \%$ respondeu que raramente os utiliza. Nenhum inquirido respondeu que nunca utiliza os materiais manipuláveis. Por fim e relativamente ao envolvimento ou não, em projetos que contemplam a integração de materiais manipuláveis, $72 \%$ respondeu que não e apenas $28 \%$ respondeu ter estado ou estar atualmente envolvido num projeto desta natureza. 


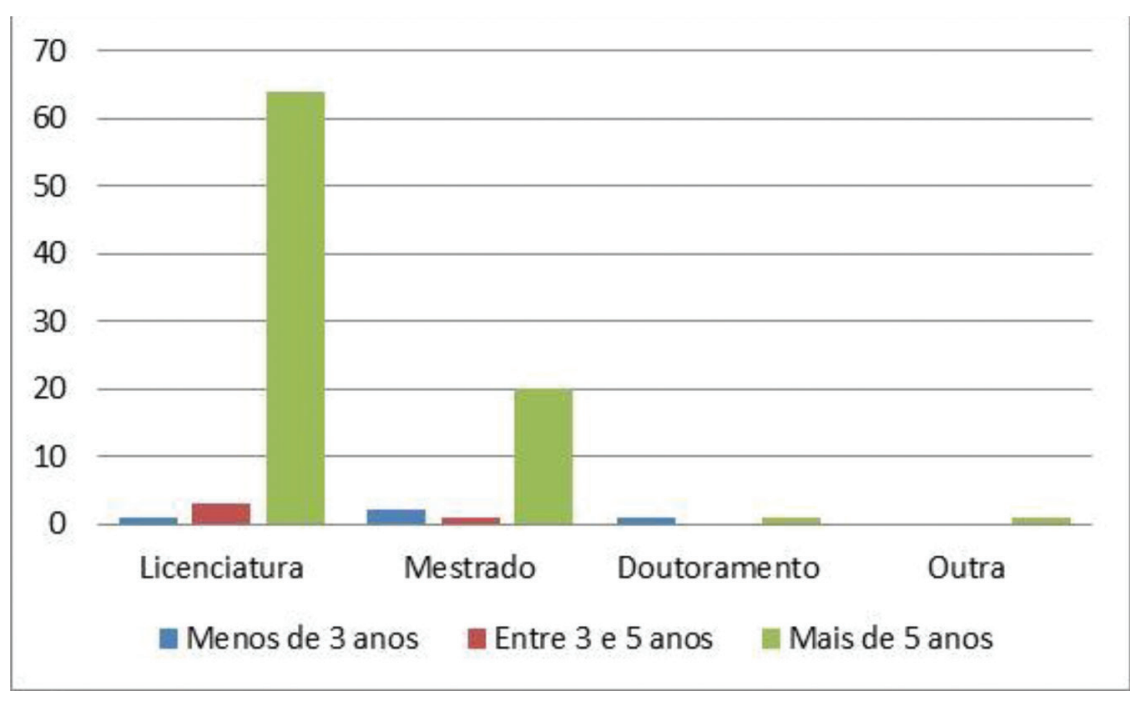

Figura 2 - Relação da Formação Académica com os anos de Experiência

\section{Conteúdos e Atividades}

A primeira análise focou-se na relação que existia entre os conteúdos matemáticos que os inquiridos gostariam de ver abordados e a atividade exercida por esses mesmos inquiridos. Nas três atividades profissionais existentes no questionário, Educador de Infância, Professor de Educação Especial e Professor do $10^{\circ} \mathrm{CEB}$, a maioria (88 \%) deu mais realce aos conteúdos relacionados com o tópico Números e Álgebra, seguido de conteúdos relacionados com o tópico Geometria. Esta tendência deve-se ao facto de os Educadores e Professores em geral verem o estudo do número, de operações com números e de técnicas de cálculo e de contagem como uma prioridade fundamental em relação a qualquer outro conteúdo matemático (Fuson, 2003).

A equipa multidisciplinar sentiu necessidade de percecionar, em que medida existia uma relação dos conteúdos (área da geometria e dos números operações) com a atividade exercida pelos inquiridos. Os resultados obtidos e apresentados na Tabela 2 indicam-nos que, os inquiridos que ocupam o cargo de Educador ou Professor valorizam mais a abordagem de conteúdos relacionados com Números e Álgebra através de uma ferramenta tecnológica digital, seguido de conteúdos relacionados com Geometria do que outros conteúdos. Os inquiridos que responderam terem como profissão "Outra" manifestaram não possuírem uma predileção específica.

\begin{tabular}{lllll}
\hline & $\begin{array}{l}\text { Educador de } \\
\text { Infância }\end{array}$ & $\begin{array}{l}\text { Professor do } \\
\mathbf{1}^{\text {O CEB }}\end{array}$ & $\begin{array}{l}\text { Professor } \\
\text { de Educação } \\
\text { Especial }\end{array}$ & Outra \\
\hline Geometria & 29 & 14 & 6 & 6 \\
\hline Números e Algebra & 47 & 24 & 12 & 6 \\
\hline
\end{tabular}

Tabela 2 - Relação entre a Profissão e os Conteúdos 
Apesar de ser interessante na definição de requisitos os resultados obtidos na Tabela 2, interessa também percecionar dentro das áreas quais os tópicos mais pretendidos que a plataforma deve conter. A Fig. 3 apresenta a relação dos tópicos da área da Geometria com a Profissão.

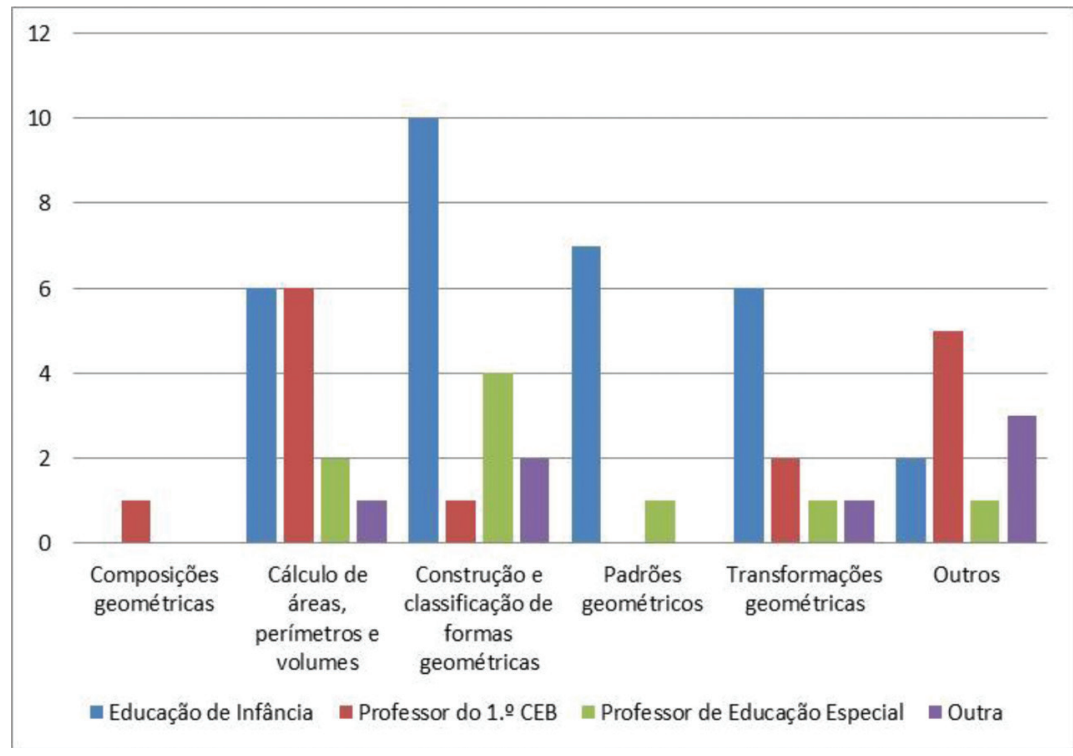

Figura 3 - Relação da Geometria vs Profissão

Da leitura efetuada à Fig. 3 é percetível a existência de uma maior apetência por parte dos Educadores de Infânia para atividades de construção e classificação deformas geométricas (10 referências). Porém, neste gráfico, ainda se verifica a ausência de respostas, por parte dos Educadores de Infância e dos Professores de Educação Especial, no que diz respeito às atividades que envolvem a Composição de Figuras Geométricas, assim como a ausência de respostas, por parte dos Professores do $10^{\circ} \mathrm{CEB}$ e dos que apresentam Outra profissão, no que concerne a atividades que envolvem Padrões Geométricos. Estes dados tornam-se relevantes na medida que evidenciam que estes grupos de Educadores não utilizam com frequência a geometria como meio para a composição de figuras. Tal facto pode dever-se a opções pedagógicas ou à escassez de ferramentas de autoria que possam facilitar a criação e o desenvolvimento destas composições.

A Fig. 4 apresenta os resultados da Relação dos Números e Álgebra com a Profissão. De salientar que, segundo estes dados, os Educadores de Infância são os profissionais que mais diversificam a utilização dos Números e Álgebra, havendo especial foco nas contagens. 


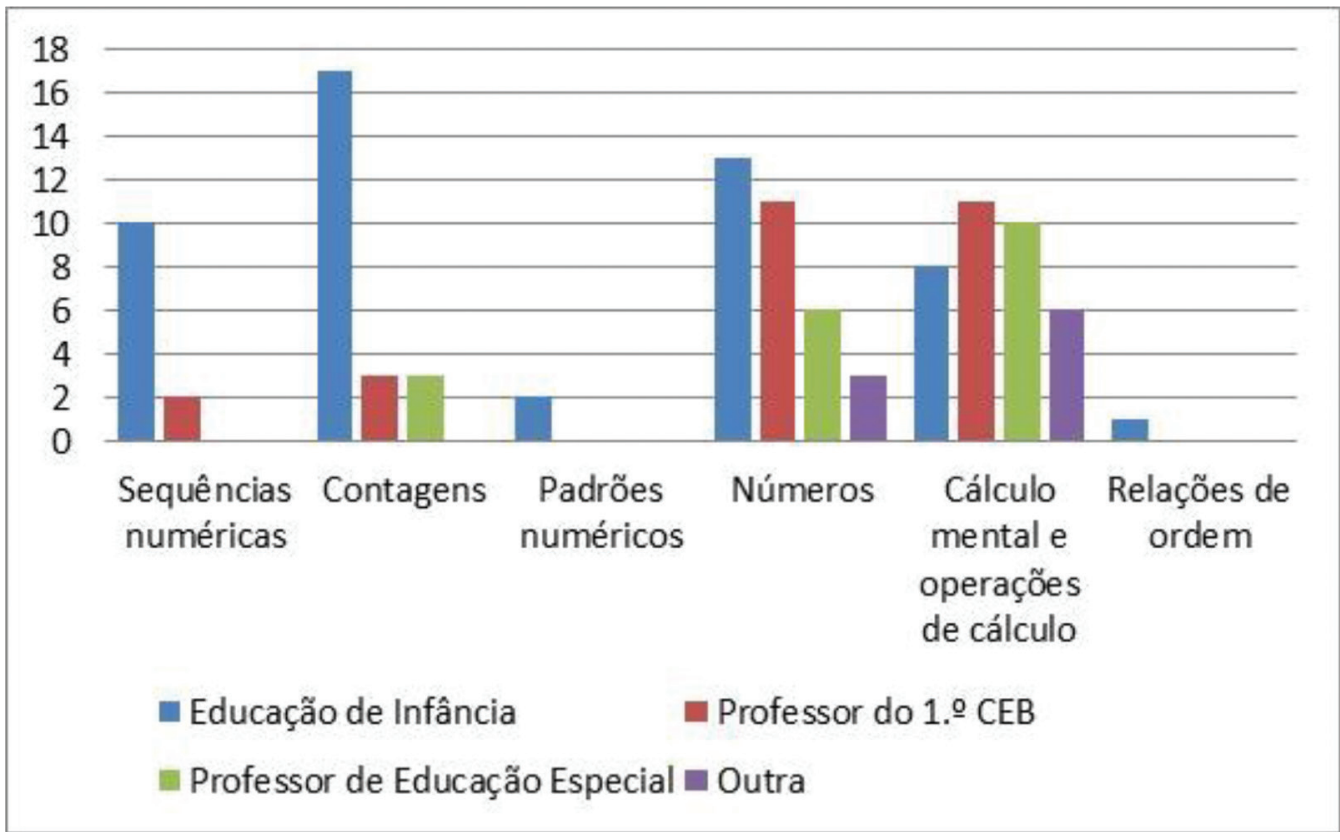

Figura 4 - Relação dos Números e Álgebra vs Profissão

\begin{tabular}{|c|c|c|c|c|c|}
\hline & $\begin{array}{l}\text { Todos os } \\
\text { dias }\end{array}$ & $\begin{array}{l}2 \text { a } 3 \\
\text { vezes por } \\
\text { semana }\end{array}$ & $\begin{array}{l}1 \text { vez por } \\
\text { semana }\end{array}$ & Raramente & Nunca \\
\hline $\begin{array}{l}\text { Associações e } \\
\text { correspondências }\end{array}$ & 5 & 5 & $\mathrm{O}$ & 1 & $\mathrm{O}$ \\
\hline Classificar & 3 & 2 & 3 & 1 & $\mathrm{O}$ \\
\hline Completar & 2 & 2 & $\mathrm{o}$ & $\mathrm{o}$ & $\mathrm{o}$ \\
\hline Construção & o & 1 & o & o & o \\
\hline Jogos & 7 & 12 & 4 & 1 & $\mathrm{O}$ \\
\hline Noções topológicas & 4 & 4 & 2 & 3 & $\mathrm{O}$ \\
\hline Ordenar & 1 & $\mathrm{O}$ & 3 & $\mathrm{O}$ & 0 \\
\hline $\begin{array}{l}\text { Questões de escolha } \\
\text { múltipla }\end{array}$ & 2 & $\mathrm{O}$ & $\mathrm{O}$ & 1 & O \\
\hline Questões diretas & $\mathrm{O}$ & $\mathrm{O}$ & $\mathrm{O}$ & 1 & $\mathrm{O}$ \\
\hline Resolução de problemas & 2 & 5 & 7 & 2 & 0 \\
\hline Seriar ou selecionar & 7 & 3 & 2 & 1 & $\mathrm{O}$ \\
\hline Testes & $\mathrm{O}$ & $\mathrm{O}$ & 1 & $\mathrm{O}$ & $\mathrm{O}$ \\
\hline
\end{tabular}

Tabela 3 - Relação da Frequência de utilização vs Atividades 
A tabela 3 relaciona a frequência com que os participantes utilizam os Materiais Manipuláveis e a influência da escolha das atividades a serem desenvolvidas com a plataforma WEBMAT.

Observando os valores presentes na Tabela 3, verificamos que a maioria dos inquiridos que utilizam os Materiais manipuláveis “Todos os dias" recorrerão, no futuro, à plataforma WEBMAT para a realização de jogos, seguido da elaboração de tarefas para seriação ou seleção. Aqueles que utilizam os Materiais Manipuláveis 2 a 3 vezes por semana irão apropriar-se das potencialidades do WEBMAT para elaboração de jogos seguido de tarefas que envolvam associações, correspondências e a resolução de problemas. A grande à utilização de jogos por parte dos Educadores (24 referências), vem reforçar as ideias apresentadas no Horizon Report Europe (Johnson et al., 2014). Está demonstrado que os estudantes que aprendem com a utilização de jogos, estão mais dispostos a colaborar e a trabalhar em grupo, devido à motivação adicional proporcionada pelos desafios colocados pelos jogos (Poy-castro, Mendaña-cuervo, \& González, 2015).

Os valores contabilizados vão diminuindo nas restantes atividades referenciadas.

A tabela 4 pretende relacionar ciclo de ensino em que o inquirido leciona, verificando se este facto influência a escolha das atividades que gostaria de desenvolver com uma ferramenta tecnológica digital.

\begin{tabular}{lllll}
\hline & $\begin{array}{l}\text { Educador } \\
\text { de Infância }\end{array}$ & $\begin{array}{l}\text { Professor do } \\
\mathbf{1}{ }^{\circ} \text { CEB }\end{array}$ & $\begin{array}{l}\text { Professor de } \\
\text { Educação Especial }\end{array}$ & Outra \\
\hline $\begin{array}{l}\text { Associações } e \\
\text { correspondências }\end{array}$ & 9 & 0 & 0 \\
\hline Classificar & 7 & 0 & 2 & 0 \\
\hline Completar & 1 & 2 & 1 & 0 \\
\hline Construção & 0 & 0 & 1 & 0 \\
\hline Jogos & 14 & 6 & 3 & 1 \\
\hline Noções topológicas & 9 & 2 & 2 & 0 \\
\hline Ordenar & 3 & 0 & 0 & 1 \\
\hline $\begin{array}{l}\text { Questões de escolha } \\
\text { múltipla }\end{array}$ & 2 & 0 & 0 & 1 \\
\hline Questões diretas & 0 & 0 & 0 & 1 \\
\hline Resolução de problemas & 6 & 3 & 6 & 1 \\
\hline Seriar ou selecionar & 9 & 0 & 3 & 1 \\
\hline Testes & 0 & 1 & 0 & 0 \\
\hline
\end{tabular}

Tabela 4 - Formação Académica vs Atividades

\section{Conclusões}

A utilização de métodos DCU confirmou que envolver o utilizador nas fases iniciais permite conhecer melhor o contexto de utilização em que aplicação será explorada e, complementarmente, definir os requisitos. Segundo Velsen, Geest, Klaassen \& Steehouder (2008), dependendo da fase em que se encontra o projeto, a avaliação pode 
servir para diferentes propósitos. Na fase inicial, em que ainda não existe nenhum software, a avaliação providência informações de apoio à tomada de decisão, numa fase intermédia e através da apresentação de protótipos, permite detetar problemas. Numa fase final, já com uma versão completa do software, permite aferir a qualidade.

Concordamos com Costa, Reis \& Loureiro (2015) que “as opções DCU devem ter por base o equilíbrio custo-benefício". O método DCU que foi aplicado neste estudo, "Análise de Requisitos do Utilizador", é um dos mais importantes e que normalmente não é utilizado. Este método, segundo Maguire (2001), comparativamente a outros métodos, necessita de mais tempo (normalmente 15 dias) para a sua implementação.

O próximo passo deste estudo será verificar, com a equipa multidisciplinar, a exequibilidade das funcionalidades (prototipagem) relativamente ao que foi solicitado em termos de conteúdos e atividades.

\section{Agradecimentos}

Agradecemos a toda a equipa da Ludomedia pelo apoio na aplicação deste estudo.

\section{Referências}

Abras, C., Maloney-krichmar, D., \& Preece, J. (2004). User-Centered Design, pp. 1-14.

Africano, D., Berg, S., Lindbergh, K., Nilbrink, F., Persson, A., Program, C. S., \& Lundholm, P. (2004). Designing Tangible Interfaces for Children 's Collaboration, pp. 853-868.

Bivar, A., Grosso, C., Oliveira, F., \& Timóteo, M. C. (2013). Programa e Metas Curriculares Matemática Ensino Básico. Lisboa: DGIDC - Ministério da Educação.

Costa, A. P. (2012). Metodologia Híbrida de Desenvolvimento Centrado no Utilizador. Universidade de Aveiro.

Costa, A. P., Loureiro, M. J., \& Reis, L. P. (2014). Do Modelo 3C de Colaboração ao Modelo 4C: Modelo de Análise de Processos de Desenvolvimento de Software Educativo. Revista Lusófona de Educação, (27), pp. 181-200.

Costa, A. P., Reis, L. P., \& Loureiro, M. J. (2014a). Desenvolvimento de Software Educativo: A Coordenação como Fator Crítico de Sucesso. RISTI - Revista Ibérica de Sistemas E Tecnologias de Informação, 1(E2), 1-18. doi:10.17013/risti.e2.1-18

Costa, A. P., Reis, L. P., \& Loureiro, M. J. (2014b). Hybrid User Centered Development Methodology: An Application to Educational Software Development. In Y. Cao, T. Väljataga, J. K. T. Tang, H. Leung, \& M. Laanpere (Eds.), Advances in Web-Based Learning - ICWL 2014 (New Horizo., Vol. 8699, pp. 243-253). Cham: Springer International Publishing. doi:10.1007/978-3-319-13296-9

Costa, A. P., Reis, L. P., \& Loureiro, M. J. (2015). Lessons Learned on Developing Educational Systems Using a Hybrid User Centered Methodology. In Á. Rocha, A. M. Correia, S. Costanzo, \& L. P. Reis (Eds.), New Contributions in Information Systems and Technologies (Advances i., pp. 213-222). Switzerland: Springer International Publishing. doi:10.1007/978-3-319-16528-8_20 
Freitas, F. M., Costa, A. P., \& Souza, F. N. De. (2015). Usability and User Experience of the WEBMAT @ Platform. In A. P. Costa, J. L. de Carvalho, P. R. Valente, \& R. N. Linhares (Eds.), Atas do $4^{\circ}$ Congresso Ibero-Americano em Investigação Qualitativa e do $6^{\circ}$ Simpósio Internacional de Educação e Comunicação (Vol. 4, pp. 13-17). Aracaju: Ludomedia.

Fuson, K. (2003). Developing mathematical power in whole number operations. In J.Kilpatrick, W. G. Martin, \& D. Schiffer (Eds.), A Research Companion for Principles and Standards for School Mathematics (pp. 69-93). Reston, Va: National Council of Teachers of Mathematics.

ISO9241. (2010). Ergonomics of human-system interaction - Part 210: Human-centred design for interactive systems. International Standards Organisation (First edit.). Geneva.

Johnson, L., Becker, S. A., Estrada, V., Freeman, A., Kampylis, P., Vuorikari, R., \& Punie, Y. (2014). The NMC Horizon Report Europe: 2014 Schools Edition. Luxembourg. doi:10.2791/83258

Kolb, D. A. (1984). Experiential Learning: experience as the source of learning and development . New Jersey: Prentice-Hall, Englewood Cliffs.

Maguire, M. (2001). Methods to support human-centred design. International Journal of Human-Computer Studies, 55(4), pp. 587-634. doi:10.1006/ijhc.2001.0503

Mao, J.-Y., Vredenburg, K., Smith, P. W., \& Carey, T. (2001). User-centered design methods in practice: a survey of the state of the art. Conference of the Centre for Advanced Studies on Collaborative Research. Toronto, Ontario, Canada: IBM Press.

Marcus, A. (2005). User-centered design in the enterprise. Interactions, 12(1), 18. doi:10.1145/1041280.1041293

Mizukami, M. da G. N. (1986). Ensino:as abordagens do processo. São Paulo: EPU.

Nesset, V., \& Large, A. (2004). Children in the information technology design process: A review of theories and their applications. Library \& Information Science Research, 26(2), pp. 140-161. doi:10.1016/j.lisr.2003.12.002

Pardo, S., Vetere, F., \& Howard, S. (2005). BROADENING STAKEHOLDER INVOLVEMENT IN UCD : DESIGNERS 'PERSPECTIVES ON CHILD-CENTRED, 1-9.

Pardo, S., Vetere, F., \& Howard, S. (2006). Teachers' involvement in usability testing with children. Proceeding of the 2006 Conference on Interaction Design and Children - IDC 'o6, 89. doi:10.1145/1139073.1139097

Ponte, J. P. Da. (2003). O ensino da matemática em Portugal: Uma prioridade educativa? O Ensino Da Matemática: Situação E Perspectivas, (i), pp. 1-28.

Poy-castro, R., Mendaña-cuervo, C., \& González, B. (2015). Diseño y evaluación de un juego serio para la formación de estudiantes universitarios en habilidades de trabajo en equipo. RISTI - Revista Ibérica de Sistemas E Tecnologias de Informação, E3(o3), pp. 71-83. doi:10.17013/risti.e3.71-83 
Preece, J., Rogers, Y., \& Sharp, H. (2002). Interaction Design: beyond human - computer interaction. Johm Wiley \& Sons.

Razón, O. C., Durán, J. G., Alonso, J., González, G., Cortés, I., \& Miranda, J. M. (2014). Cómo Guiar a las Pymes en la Mejora de Procesos Software. Revista Ibérica de Sistemas E Tecnologias de Informação, E1(03), pp. 17-30. doi:10.4304/risti.e1.17-30

Robert, A. D., \& Bouillaguet, A. (1997). L'analyse de contenu (Presses un.).

Scaife, M., Rogers, Y., Aldrich, F., \& Davies, M. (1997). Designing for or designing with? Informant design for interactive learning environments. Proceedings of the SIGCHI Conference on Human Factors in Computing Systems - CHI '97, pp. 343-350. doi:10.1145/258549.258789

Scanlon, E., Mcandrew, P., \& Shea, T. O. (2015). Designing for Educational Technology to Enhance the Experience of Learners in Distance Education : How Open Educational Resources, Learning Design and Moocs Are Influencing Learning. Journal of Interactive Media in Education, 1(6), 1-9. doi: http://doi.org/10.5334/jime.al

Soloway, E., Guzdial, M., \& Hay, K. E. (1994). Learner-centered design: the challenge for $\mathrm{HCI}$ in the 21st century. Interactions, 1(2), pp. 36-48.

Souza, F. N. de, Costa, A. P., \& Moreira, A. (2011). webQDA. Aveiro: Centro de Investigação Didática e Tecnologia na Formação de Formadores da Universidade de Aveiro e Esfera Crítica. Retrieved from www.webqda.com

The Role of Children in the Design of New Technology (2002). Behaviour and Information Technology (BIT), 21(1), 1-25.

UNESCO. (2014). Tecnologias para a transformação da educação: experiências bem sucedidas e expectativas.

Van Velsen, L., Van Der Geest, T., Klaassen, R., \& Steehouder, M. (2008). User-centered evaluation of adaptive and adaptable systems: a literature review. The Knowledge Engineering Review, 23(03), pp. 261-281. doi:10.1017/So269888908001379

Víctor López Simó, \& Casulleras, R. P. (2013). Identificación de las Dificultades de los Estudiantes de Secundária en la Lectura de las Imágenes Científicas Digitales e Interactivas (pp. 1984-1991). Girona: IX Congreso Internacional sobre Investigación em Didáctica de las Ciencias. 